\title{
The Galaxy's Eating Habits
}

\author{
M. E. Putman ${ }^{1}$ \\ Center for Astrophysics and Space Astronomy, University of Colorado, \\ Boulder, CO 80309-0389, USA \\ ${ }^{1}$ Hubble Fellow \\ C. Thom, B. K. Gibson \\ Centre for Astrophysics 83 Supercomputing, Swinburne University, \\ Hawthorn, VIC 3122, Australia \\ L. Staveley-Smith \\ Australia Telescope National Facility, CSIRO, P.O. Box 76, Epping, \\ NSW 1710, Australia
}

\begin{abstract}
The possibility of a gaseous halo stream which was stripped from the Sagittarius dwarf galaxy is presented. The total mass of the neutral hydrogen along the orbit of the Sgr dwarf in the direction of the Galactic Anti-Center is $4-10 \times 10^{6} \mathrm{M}_{\odot}$ (at $36 \mathrm{kpc}$, the distance to the stellar debris in this region). Both the stellar and gaseous components have negative velocities in this part of the sky, but the gaseous component extends to higher negative velocities. We suggest this gaseous stream was stripped from the main body of the dwarf $0.2-0.3 \mathrm{Gyr}$ ago during its current orbit after a passage through a diffuse edge of the Galactic disk with a density $>10^{-4} \mathrm{~cm}^{-3}$. The gas would then represent the dwarf's last source of star formation fuel and explains how the galaxy was forming stars 0.5-2 Gyr ago.
\end{abstract}

\section{Introduction}

Our Galaxy is in the process of consuming its small neighbors. Though this may sound rather grim, it is its only source of fuel and has allowed our Galaxy to grow into the large spiral it is today. One of the closest examples of a recognizable accreting satellite is the Sagittarius dwarf galaxy $(28 \mathrm{kpc}$; hereafter Sgr dwarf; Ibata et al. 1994). The evidence continues to accumulate that contiguous streams of leading and trailing stellar debris are being pulled from the Sgr dwarf as it spirals into the Milky Way (e.g., Newberg et al. 2003), with the recent 2MASS data tracing this trail around the majority of the Galaxy (Majewski et al. 2003; hereafter M03). The stars associated with the Sgr dwarf span a wide range of ages, with the youngest population between 0.5 - 3 Gyr old (Layden \& Sarajedini 2000; Dolphin 2002; M03). This indicates that within the past Gyr, and during its current orbit about the Galaxy, the Sgr dwarf was forming stars and had a source of star formation fuel. 
Neutral hydrogen is a principal source of star formation fuel for a galaxy. Galaxies which contain HI are commonly currently forming stars (e.g., Lee et al. 2002) and those without detectable HI tend to have primarily an older stellar population and thus appear to have exhausted their star formation fuel (e.g., Gavazzi et al. 2002). This is evident in the dwarf galaxies of the Local Group. The stars in the Local Group dwarfs vary from being almost entirely ancient (> 10 Gyr; e.g., Ursa Minor) to a number of systems which are actively forming stars (e.g., WLM, Phoenix, LMC). The majority of the Local Group galaxies with HI have formed stars within the past $2 \mathrm{Gyr}$ and those with no evidence for recent star formation do not contain detectable HI (e.g., Mateo 1998; Dolphin 2002; Bouchard et al. 2003).

HI observations of the central region of the Sgr dwarf $\left(\alpha, \delta=19^{h} 00^{m}\right.$, $\left.-30^{\circ} 25^{\prime} ; \mathrm{J} 2000\right)$ indicate that our closest satellite galaxy does not currently contain a significant amount of star formation fuel $\left(\mathrm{M}_{\mathrm{HI}}<1.5 \times 10^{4} \mathrm{M}_{\odot}(3 \sigma)\right.$; Koribalski, Johnston \& Otrupcek 1994). The search for HI associated with the Sgr dwarf was continued by Burton \& Lockman (1999); but they also found no associated gas over $18 \mathrm{deg}^{2}$ around the core of the Sgr dwarf with limits of $\mathrm{M}_{\mathrm{HI}}<7 \times 10^{3} \mathrm{M}_{\odot}(3 \sigma)$. These results are surprising considering the Sgr dwarf was forming stars within the last Gyr. Since the orbit of the Sgr dwarf is approximately 0.7 Gyr (Ibata \& Lewis 1998), one might expect to find this fuel stripped along the dwarfs orbit, possibly at a similar location to the stellar trail. The trailing stellar tidal tail of the Sgr dwarf has recently been found to extend for over $150^{\circ}$ across the South Galactic Hemisphere with a mean distance between 20 to $40 \mathrm{kpc}$ from the Sun (M03). Here we present HI data from HIPASS (HI Parkes All-Sky Survey ${ }^{1}$ ) along the entire Sgr dwarf galaxy orbit to investigate the possibility that a gaseous Sgr trail is also present. The gas detected represents a potential method of tracing the history, make-up, and classification of the Sgr dwarf galaxy, as well as the construction of our Galaxy.

\section{Observations}

The neutral hydrogen data are from the HI Parkes All--Sky Survey (HIPASS) reduced with the MINMED5 method (see Putman et al. 2003a for a description of this reduction method). HIPASS is a survey for HI in the Southern sky, extending from the South celestial pole to Decl. $=+25^{\circ}$, over velocities from -1280 to $+12700 \mathrm{~km} \mathrm{~s}^{-1}$ (Barnes et al. 2001). The survey utilized the 64$m$ Parkes radio telescope, with a focal-plane array of 13 beams arranged in a hexagonal grid, to scan the sky in $8^{\circ}$ zones of Decl. with Nyquist sampling. The MINMED5 reduced HIPASS data has a spatial resolution of $15.5^{\prime}$ and a spectral resolution, after Hanning smoothing, of $26.4 \mathrm{~km} \mathrm{~s}^{-1}$. For extended sources, the RMS noise is $10 \mathrm{mJy}$ beam $^{-1}$ (beam area $243 \operatorname{arcmin}^{2}$ ), corresponding to a brightness temperature sensitivity of $8 \mathrm{mK}$. The northern extension of the survey, from $+2^{\circ}$ to $+25^{\circ}$, was only recently completed and is presented for the first time here. The noise in these cubes is slightly elevated compared to the

\footnotetext{
${ }^{1}$ The Parkes Telescope is part of the Australia Telescope which is funded by the Commonwealth of Australia for operation as a National Facility managed by CSIRO.
} 
southern data (11 mK vs. $8 \mathrm{mK}$ ). This may be due to a combination of low zenith angles during these observations and an inability to avoid solar interference as effectively. Integrated intensity maps of the high positive and negative velocity gas (generally $\left|\mathrm{v}_{\mathrm{lsr}}\right|>80 \mathrm{~km} \mathrm{~s}^{-1}$, as long as the gas was clearly separate from Galactic emission) were made for the $24 \mathrm{deg}^{2}$ cubes which lie along the Sgr orbit. The positive velocity cubes had very little emission in them, so we concentrated on the negative velocity cubes. The noise at the edges of the negative velocity maps were blanked within AIPS and the images were then read into IDL to create the map of the entire orbit shown in Figure 1. At 20-40 kpc the MINMED5 reduced HIPASS data has a sensitivity to clouds of gas with $\mathrm{M}_{\mathrm{HI}}>80-320$ $\mathrm{M}_{\odot}\left(\Delta \mathrm{v}=25 \mathrm{~km} \mathrm{~s}^{-1} ; 3 \sigma\right)$.

\section{Results}

The large scale HI map which includes all of the high negative velocity gas along the orbit of the Sgr dwarf is shown in Figure 1. The gas attributed to the Magellanic Stream and Galactic Center is labeled. The stream of M giants presented by M03 has quite a broad width (commonly several degrees) along this orbit. The only region which has both gas and stars at negative velocities is between $\alpha=2$ to $5.5 \mathrm{~h}$ and $\delta=0$ to $30^{\circ}$, or $\ell \approx 155$ to $195^{\circ}$ and $b \approx-5$ to $-50^{\circ}$. This high velocity $\mathrm{HI}$ gas was previously identified as part of the anticenter complexes (ACHV and ACVHV; Wakker \& van Woerden 1991), but its relationship to the Sgr dwarf was not previously noted. This gas extends over the velocity range of -380 to $-125 \mathrm{~km} \mathrm{~s}^{-1}$ (LSR), with the gas clouds orientated along the orbit of the Sgr dwarf $(\alpha \sim 2-4.5 \mathrm{~h})$ in the velocity range -380 to -180 $\mathrm{km} \mathrm{s}^{-1}$ (see the channel maps in Putman et al. 2003b). The distinct filament extending vertically across the Sgr orbit at $\alpha \sim 5 \mathrm{~h}$ is in the velocity range of -245 to $-75 \mathrm{~km} \mathrm{~s}^{-1}$. All of the gas shown in this region would have a mass of $10^{7} \mathrm{M}_{\odot}$ at $36 \mathrm{kpc}$, the approximate distance to the stars in the Sgr stream (M03; Ibata et al. 2001). If only the stream of clouds orientated along the orbit of the Sgr dwarf is considered, the HI mass is $4 \times 10^{6} \mathrm{M}_{\odot}$. The gas has peak column densities on the order of $10^{20} \mathrm{~cm}^{-2}$ at the $15.5^{\prime}$ resolution of HIPASS and extends to the column density limits of the data $\left(5 \sigma \sim 3 \times 10^{18} \mathrm{~cm}^{-2} ; \Delta \mathrm{v}\right.$ $\left.=25 \mathrm{~km} \mathrm{~s}^{-1}\right)$. The carbon stars associated with the Sgr dwarf at this position have velocities between -140 to $-160 \mathrm{~km} \mathrm{~s}^{-1}$ (LSR; e.g., Dinescu et al. 2002). The negative velocity gas clouds at $(\alpha, \delta) \sim 15.25 \mathrm{~h},-19.5^{\circ}$ represent Complex $\mathrm{L}$ and are in a region of the Sgr stellar stream that has positive velocity stars.

\section{Discussion}

The present orbit of the Sgr dwarf is estimated to be 0.7 Gyr (Ibata \& Lewis 1998). Using this orbit, the core of the Sgr dwarf was at the position of the HI complex noted here approximately 0.2 to $0.3 \mathrm{Gyr}$ ago. It would make sense if the gas was part of the Sgr dwarf within that timescale considering the age of the Sgr dwarf stellar population (0.5 - 3 Gyr; Layden \& Sarajedini 2000; Dolphin 2002; M03). These gas clouds are along a sightline relatively close to the plane of our Galaxy, and at this position the Sgr dwarf stellar debris is approximately $45 \mathrm{kpc}$ from the Galactic Center (M03). This distance is significantly beyond 
the typical radius quoted for our Galaxy $(\sim 26 \mathrm{kpc})$, however it is possible that an extended ionized disk exists (e.g., Savage et al. 2003), as found in other systems (Bland-Hawthorn, Freeman \& Quinn 1997). The passage through an extended disk of our Galaxy, in addition to the tidal forces already obviously at work as evident from the stellar tidal stream, might have been enough to disrupt the $\mathrm{HI}$ in the core of the galaxy and cause the dwarf to lose all remaining star formation fuel. The gas will be stripped from a galaxy if $\rho_{I G M} v^{2}>\sigma^{2} \rho_{\text {gas }} / 3$ (Mori \& Burkert 2000; Gunn \& Gott 1972). We can use this equation to estimate the density needed at the edge of the disk to strip the gas from the core of the Sgr dwarf. We use a tangential velocity of $280 \mathrm{~km} \mathrm{~s}^{-1}$ for the Sgr dwarf and a velocity dispersion of $11.4 \mathrm{~km} \mathrm{~s}^{-1}$ (Ibata et al. 2003; Ibata \& Lewis 1998). If the column densities and size of the HI distribution in the core of the Sgr dwarf were on the order of $5 \times 10^{20} \mathrm{~cm}^{-2}$ (averaged over the core) and $1 \mathrm{kpc}$, the typical $\rho_{\text {gas }}$ is $0.16 \mathrm{~cm}^{-3}$. An extended disk density greater than $3 \times 10^{-4} \mathrm{~cm}^{-3}$ is then needed to strip the gas via ram pressure stripping. Based on previous estimates of the density of the Galactic halo, this density would be easily achieved in the plane of the Galaxy, $20 \mathrm{kpc}$ from the currently observed edge of the disk (e.g., Sembach et al. 2003; Maloney 2003). Moore \& Davis (1994) proposed a similar model to create the Magellanic Stream.

The gas that was most likely once part of the Sgr dwarf is the stream of clouds orientated along the orbit of the Sgr dwarf between $\alpha=2-4.5 \mathrm{~h}$ and $\mathrm{v}_{\mathrm{LSR}}=-380$ to $-180 \mathrm{~km} \mathrm{~s}^{-1}$ (Figure 1). At $36 \mathrm{kpc}$ from the sun this HI gas has a mass of $4 \times 10^{6} \mathrm{M}_{\odot}$. This gas is at higher negative velocities than the carbon stars with velocity determinations in this region, but this is not unexpected with the addition of ram pressure forces to the tidal. If the filament extending perpendicular to the orbit is included as once being part of the Sgr dwarf, the total $\mathrm{HI}$ mass goes up to $10^{7} \mathrm{M}_{\odot}$. A typical total HI mass for a dwarf galaxy is on the order of $10^{7}$ to $10^{8} \mathrm{M}_{\odot}$ (Grebel et al. 2003), so this gas may represent anywhere from the majority to $10 \%$ of the total amount of gas once associated with the Sgr dwarf. Using a total Sgr dwarf mass of $5 \times 10^{8} \mathrm{M}_{\odot}(\mathrm{M} 03)$, this gas represents $1-2 \%$ of its total mass. The Sgr dwarf most likely originally had more than $10^{6}-10^{7} \mathrm{M}_{\odot}$ of neutral hydrogen associated with it, as the outer gaseous component would have been the first thing stripped from our closest satellite (e.g., Yoshizawa \& Noguchi 2003). This outer gas would have had column densities between $10^{18}-10^{19} \mathrm{~cm}^{-2}$ and has most likely either already dispersed or been ionized, although remnants of this gas may be present as small HVCs along the Sgr orbit. Since there is currently no HI associated with the core of the Sgr dwarf (Koribalski et al. 1994), and the dwarf has stars which are 0.5 - 3 Gyr old, the HI gas presented here most likely represents the high column density gas from the core of the Sgr dwarf which was finally stripped when the dwarf passed through the medium in the extended Galactic disk. This is supported by the relatively high peak column densities of the HI gas currently $\left(\sim 10^{20} \mathrm{~cm}^{-2}\right)$. For the Sgr HI gas to survive for $0.2-0.3 \mathrm{Gyr}$ (the time since the last passage of the core Sgr dwarf), not to mention the Magellanic Stream which is thought to be $>0.5$ Gyr old, the gas should either be confined by an existing halo medium or associated with significant amounts of dark matter.

The association of HI gas with the Sgr stellar stream suggests that the dwarf spheroidal classification for the Sagittarius galaxy may need to be reconsidered. It also suggests that slightly offset HI and stellar streams may be a common fea- 


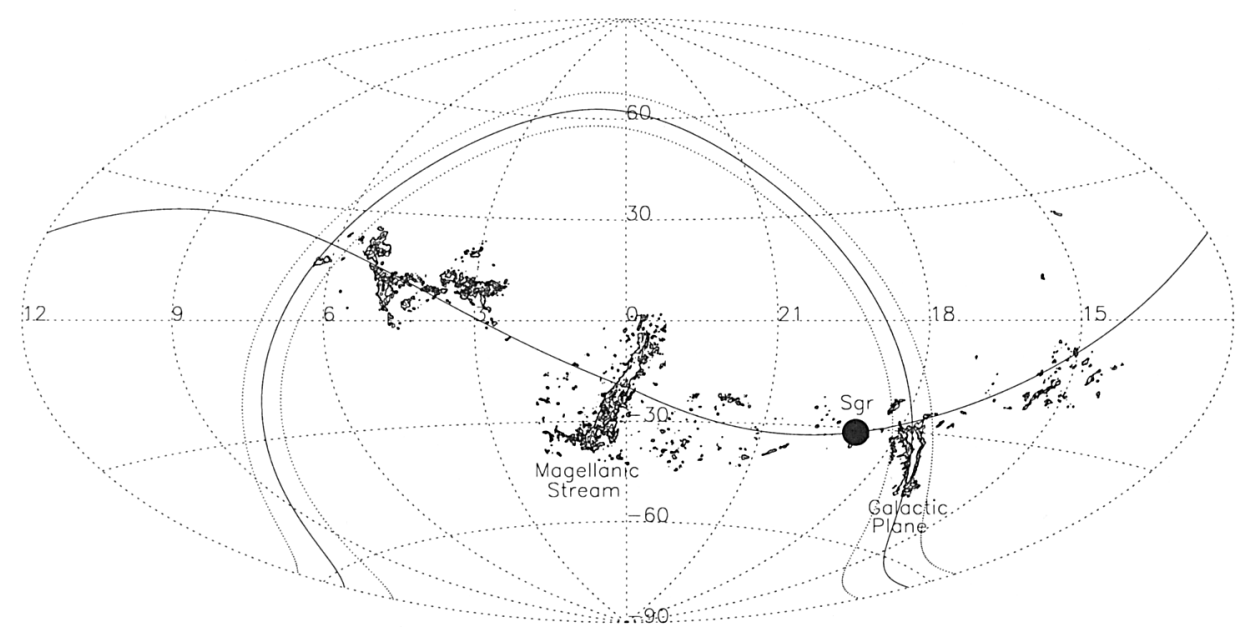

Figure 1. The negative high-velocity HI $\left(\sim-85\right.$ to $-400 \mathrm{~km} \mathrm{~s}^{-1}$; LSR) along the orbit of the Sgr dwarf galaxy in Celestial coordinates. The current position of the Sgr dwarf is shown by the solid point, and the orbit of the Sgr dwarf (Ibata \& Lewis 1998) is plotted as the solid line through this point. The negative velocity gas attributed to the Magellanic Stream and Galactic Center is labeled, and the Galactic Plane is indicated by the solid line with the two dotted lines on each side representing $b=+5^{\circ}$ and $-5^{\circ}$. The negative velocity carbon stars extend from approximately $\alpha, \delta=0^{h},-20^{\circ}$ to $12^{h}, 20^{\circ}$ along the Sgr orbit (Ibata et al. 2001). Contours represent column density levels of $0.5,1.0,5.0$, and $10.0 \times 10^{19} \mathrm{~cm}^{-2}$.

ture of disrupted satellites in the Galactic halo. With the accretion of this Sgr HI stream, the Magellanic Stream (Putman et al. 2003), Complex C (Wakker et al. 1999), and possibly other HVCs, there is ample fuel for our Galaxy's continuing star formation and understanding the distribution of stellar metallicities (e.g., the G-dwarf problem is not a problem). Determining the metallicity, distance, and ionization properties of the HI gas presented here will aid in confirming if this HI gas was indeed stripped from the Sgr dwarf during its current orbit. Since we propose this is the last gas from the core of the Sgr dwarf, we will also look for molecular gas and dust associated with these clouds and complete simulations to determine the detailed parameters of gaseous stripping from the Sgr dwarf.

Acknowledgments. We would like to thank Phil Maloney, Steve Majewski and Geraint Lewis for useful discussions. M.E.P. acknowledges support by NASA through Hubble Fellowship grant HST-HF-01132.01 awarded by the Space Telescope Science Institute, which is operated by AURA Inc. under NASA contract NAS 5-26555. B.K.G. acknowledges the support of the Australian Research Council, through its Large Research Grant and Discovery Project schemes. 


\section{References}

Barnes, D.G. et al. 2001, MNRAS, 322, 486

Bland-Hawthorn, J., Freeman, K.C., \& Quinn, P.J. 1997, ApJ, 490, 143

Bouchard, A. et al. 2003, AJ, in preparation

Burton, W.B. \& Lockman, F.J. 1999, A\& A, 349, 7

Dinescu, D.I. et al. 2002, ApJ, 575, 67

Dolphin, A.E. 2002, MNRAS, 332, 91

Gavazzi, G., Bonfanti, C., Sanvito, G., Boselli, A., Scodeggio, M. 2002, ApJ, 576,135

Grebel, E.K., Gallagher, J.S. III, \& Harbeck, D. 2003, AJ, 125, 1926

Gunn, J.E. \& Gott, J.R., III 1972, ApJ, 176, 1

Ibata, R.A., Gilmore, G., \& Irwin, M.J. 1994, Nature, 370, 194

Ibata, R.A. \& Lewis, G.F. 1998, ApJ, 500, 575

Ibata, R.A., Lewis, G.F., Irwin, M., Totten, E. \& Quinn, T. 2001, 551, 294

Ibata, R.A., Gilmore, G., Irwin, M., Lewis, G., Wyse, R. \& Suntzeff, N. 2003, ApJ, in preparation

Koribalski, B, Johnston, S. \& Otrupcek, R. 1994, MNRAS, 270, 43

Layden, A.C. \& Sarajedini, A. 2000, AJ, 119, 1760

Lee, J.C., Salzer, J.J., Impey, C., Thuan, T.X., \& Gronwall, C. 2002, AJ, 124, 3088

Majewski, S.R., Skrutskie, M.F., Weinberg, M.D. \& Ostheimer, J.C. 2003, ApJ, 599, 1082 (M03)

Maloney, P.R. 2003, in The IGM/Galaxy Connection, eds. J.L. Rosenberg \& M.E. Putman, Kluwer Academic Publishers, 299

Mateo, M. 1998, ARA\&A, 36, 435

Moore, B. \& Davis, M. 1994, 270, 209

Mori, M. \& Burkert, A. 2000, ApJ, 538, 559

Newberg, H.J. et al. 2003, ApJ, 596, L191

Putman, M.E., Staveley-Smith, L., Freeman, K.C., Gibson, B.K. \& Barnes, D.G. 2003a, ApJ, 586, 170

Putman, M.E., Thom, C., Gibson, B.K., \& Staveley-Smith, L. 2003b, ApJL, submitted (astro-ph/0311053)

Savage, B.D., Sembach, K. R., Wakker, B. P., Richter, P., Meade, M., Jenkins, E. B., Shull, J. M., Moos, H. W., \& Sonneborn, G. 2003, ApJS, 146, 125

Sembach, K.R., Wakker, B. P., Savage, B. D., Richter, P., Meade, M., Shull, J. M., Jenkins, E. B., Sonneborn, G., \& Moos, H. W. 2003, ApJS, 146, 165

Wakker, B.P. et al. 1999, Nature, 402, 388

Wakker, B.P. \& van Woerden, H. 1991, A\&A, 250, 509

Yoshizawa, A.M. \& Noguchi, M. 2003, MNRAS, 339, 1135 